\title{
Immune senescence and cardiomyopathy associated with obesity
}

\begin{abstract}
Obesity, diabetes, and metabolic syndrome are common comorbidities in patients with heart failure with preserved ejection fraction. The molecular mechanisms by which these comorbidities promote LV diastolic dysfunction are complex. This review summarizes research performed in mice showing that excessive saturated fatty acid intake causes LV diastolic dysfunction by accelerating immune senescence and increasing cardiomyocyte membrane stiffness, as well as discussing preventive strategies.
\end{abstract}

Keywords: Osteopontin - Immune senescence - Obesity-induced cardiomyopathy • Diastolic dysfunction - Monounsaturated fatty acids - Sirt- 1 - Membrane fluidity

\section{OPN, A Biomarker of Aging}

Chronic inflammation is involved in many age-associated chronic disorders, such as diabetes, cardiovascular disease (CVD), and heart failure, a phenomenon known as "inflamm-aging". Discovery of targets that suppress "inflamm-aging" could lead to the development of anti-aging drugs. We have focused on osteopontin (OPN), since a high circulating OPN level is associated with an increased risk of cardiovascular death or heart failure [1]. Typically, the blood level of OPN increases with age. Surprisingly, healthy centenarians have lower OPN levels than healthy controls in their $70 \mathrm{~s}$ [2]. This observation suggests that lower OPN levels are associated with successful aging. OPN activates the immune system and plays an important role in the wound healing process. After myocardial infarction, OPN is produced by M2-like macrophages, and it induces phagocytosis of dead cells and reparative fibrosis that promotes scar formation at the infarct site [3]. However, sustained and uncontrolled OPN production causes chronic systemic low-grade inflammation, leading to development of various diseases associated with aging. Thus, OPN is a potential biomarker and target for personalized antiaging therapies.

\section{Senescence-associated T cells are a major source of OPN in the elderly}

In elderly persons, OPN is constitutively secreted by senescent CD4 $\mathrm{T}$ cells [4]. The proportion of $\mathrm{CD} 4 \mathrm{~T}$ cells showing high surface expression of programmed cell death 1 (PD-1) increases with age. These PD-1hi CD4 $T$ cells are senescent cells, not functionally inactivated cells. As their senescence-associated secretory phenotype (SASP), senescent CD4 T cells show high secretion of OPN. On the other hand, senescent $\mathrm{CD} 4 \mathrm{~T}$ cells lose their ability to maximize antipathogenic immune responses, while suppressing nonessential immune responses. Accordingly, the immune system becomes unbalanced as the proportion of senescent $T$ cells increases. Senescent $T$ cells showing high secretion of OPN are known as "senescence-associated T cells", and these cells are a major source of circulating OPN in elderly persons. Because the increase of senescence-associated $\mathrm{T}$ cells unbalances the immune system, it is considered to be the basis of immune senescence, which is
Motoaki Sano*, Kohsuke Shirakawa, Tsunehisa Yamamoto

Department of Cardiology, Keio University School of Medicine, Japan

*Author for correspondence: Motoaki Sano

Email: msano@a8.keio.jp

Received date: August 12, 2019 Accepted date: August 26, 2019

Published date: September 02, 2019 
characterized by impairment of acquired immunity, a predisposition to inflammation, increased susceptibility to autoimmune disease.

\section{Visceral adiposity accelerates immune senescence}

Visceral adiposity accelerates aging by enhancing inflammation and increases the risk of CVD and heart failure. We have found that $\mathrm{T}$ cells undergo cellular senescence in obese visceral adipose tissue (VAT) [5,6]. When mice were fed either a high fat diet (HFD) or a control diet containing less fat, the HFD caused weight gain, fat deposition in VAT, impaired glucose tolerance, and insulin resistance. F $4 / 80+\mathrm{CD} 11 \mathrm{~b}+$ macrophages are increased in obese VAT, indicating a shift of macrophage polarity to the proinflammatory phenotype. Macrophages were localized to crown-like structures in VAT, which are a histologic hallmark of chronic inflammation, and circulating OPN levels were increased in HFD-fed obese mice. Figure 1 shows identification of cells undergoing senescence in the VAT of obese mice by detection of senescence-associated $\beta$-galactosidase activity. The senescence-associated $\beta$-galactosidase-positive cells were found to be $\mathrm{T}$ cells invading the region around necrotic adipocytes. The HFD induced accumulation of PD-1hi CD4+ T cells in VAT, and more than half of VAT CD44 high CD4+ $T$ cells expressed PD-1 in 18-week-old HFD-fed mice. Senescent $T$ cells in VAT showed the same phenotypic profile as senescent $\mathrm{T}$ cells from aged mice, including predominance of OPN production, positivity for senescence-associated $\beta$-galactosidase, and elevated $\gamma-\mathrm{H} 2 \mathrm{AX}$ expression, indicating greater genetic stress. These results suggested that visceral adiposity is linked to $T$ cell senescence independently of aging. We found that CD153 expression defines a unique PD-1hi CD4+ $T$ cell population with senescent features and high OPN secretion. These cells did not exist in the adipose tissue of young lean mice. To confirm whether CD153+ PD1hi CD4+ T cells produce OPN in the VAT of HFDfed obese mice, we investigated EGFP-Spp1 knockin reporter mice fed the HFD. In this mouse model, cells with high Spp1 gene transcriptional activity can be identified as GFP-positive cells. Among CD4+ T cells in the VAT, GFP was almost exclusively expressed by the PD-1hi cell population, in which expression of GFP and CD153 were closely correlated. Thus, CD153+PD-1hi CD4+T cells are the main source of OPN in the VAT of HFD-fed mice, which implies that $T$ cell senescence is a common mechanism underlying diseases associated with obesity and aging (Figure 2).

\section{Anti-aging vaccine}

In order to eliminate senescence-associated $\mathrm{T}$ cells, a vaccine was developed by targeting an antigen specifically expressed on the surface of these $T$ cells. When this vaccine was administered to obese diabetic mice and induced the production of cytotoxic antibodies targeting senescence-associated $\mathrm{T}$ cells, the number of these $\mathrm{T}$ cells in VAT was decreased, along with improvement of VAT inflammation and glucose tolerance. This anti-aging vaccine is a potential tool for promotion of productive aging.

\section{Differences between obese persons with/without obesity-associated diseases}

Many overweight or obese persons are healthy. We investigated whether different types of dietary fat influenced the susceptibility of $\mathrm{T}$ cells to senescence. Mice were fed either a saturated fatty acid-rich, lardbased HFD or a monounsaturated fatty acid-rich, olive oil-based HFD (Figure 3). These two diets had the same total calories and the same fat content, resulting in equal

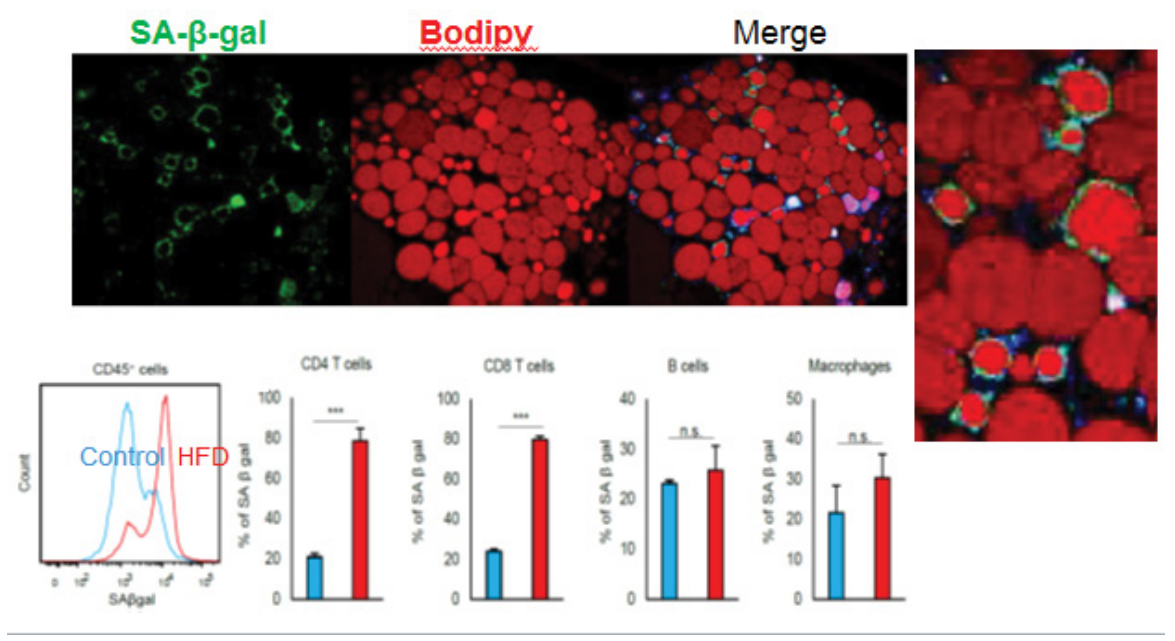

Figure 1:T cells undergoing cellular senescence in visceral adipose tissue of young obese mice. 
Obesity $\checkmark$

T cell senescence $\checkmark$

\section{Inflammation} $\checkmark$ Osteopontin

\section{CVD/heart failure/death}

Figure 2: $T$ cell senescence is a mechanism common to diseases associated with obesity and aging.
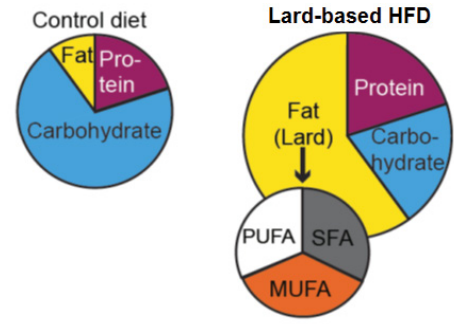

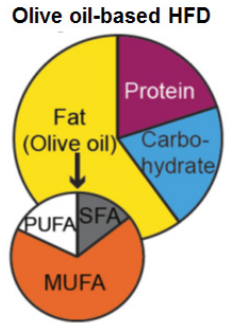

Figure 3: Saturated fatty acid-rich vs. monounsaturated fatty acid-rich HFD.

levels of obesity. Compared to mice fed the lard-based HFD, mice fed the olive oil-based HFD had less VAT inflammation and lower insulin levels. In mice receiving the olive oil-based HFD, senescence-associated T cells were not detected in VAT and the circulating OPN level remained low. Based on these findings, we concluded that the appearance of senescence-associated $\mathrm{T}$ cells in VAT, chronic VAT inflammation, and high circulating OPN levels are induced by a saturated fatty acid-rich HFD, but are less likely to occur with a monounsaturated fatty acid-rich HFD.

\section{Decrease of unsaturated membrane phospholipids is correlated with diastolic dysfunction}

The mechanisms leading to cardiac dysfunction are multifactorial, and may include generation of excess ROS by the mitochondria due to fatty acid overload or accumulation of harmful lipid intermediate metabolites such as ceramide and diacylglycerol. However, whether changes in the fatty acid composition of membrane phospholipids are involved in the development of cardiac dysfunction associated with fatty acid overload has not been examined.
In mice, intake of a lard-based HFD caused cardiac hypertrophy, interstitial fibrosis, and cardiomyocyte death, while such changes were rare in mice fed an olive oil-based HFD [7]. The lard-based HFD caused diastolic dysfunction, while the olive oil-based HFD did not. Both HFDs caused a similar increase in the expression of various genes involved in transcriptional regulation of mitochondrial function (Tfam, Ppara, Pgc1 $\alpha$, and Nrf1) and TG turnover (Pnpla2/Atgl, and Dgat1), as well as PPAR $\alpha$ target genes involved in fatty acid uptake and mitochondrial oxidation (Cpt1, Cd36, and Acsl1). Both HFDs caused a similar increase of the total TG, DAG, and ceramide content in cardiomyocytes. However, there was a difference in the fatty acid composition of membrane phospholipids. The lard-based HFD increased the SFA/MUFA ratio of membrane phospholipids, while this change was not observed in mice receiving the olive oil-based HFD. Generally, membrane fluidity decreases when the SFA/ MUFA ratio of membrane phospholipids increases, leading to impairment of membrane protein function. Thus, the lard-based HFD increased the SFA/MUFA ratio in cardiomyocytes, leading to LV remodeling and diastolic dysfunction (Figure 4).

\section{Sirt1 prevents membrane phospholipid unsaturation and diastolic dysfunction due to saturated fatty acid overload}

The lard-based HFD was associated with reduced Sirt1 expression in cardiomyocytes, while the olive oil-based HFD was not. Therefore, we examined the role of cardiac Sirt1 in cardiomyocyte-specific Sirt1-KO mice [8]. In mice fed a lard-based HFD, we found that Sirt1 deficiency in cardiomyocytes decreased the expression of SCD-1 (a rate-limiting enzyme for synthesis of MUFA from SFA), increased the membrane SFA/MUFA ratio, and exacerbated LV diastolic dysfunction. Next, we

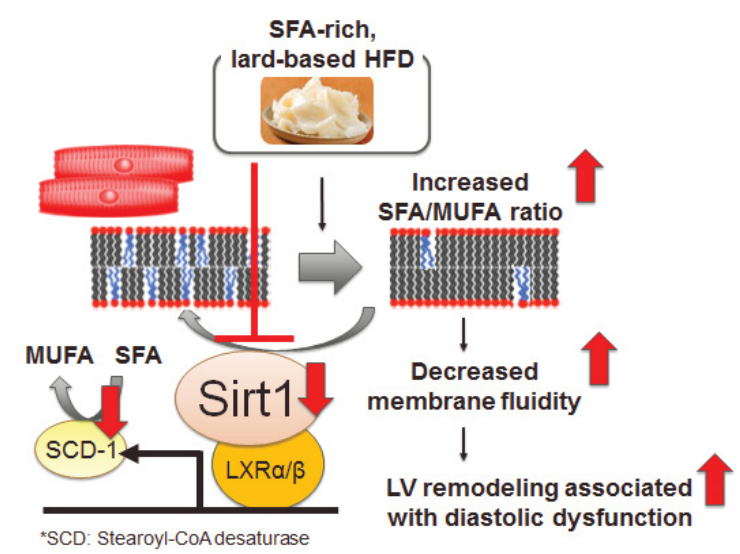

Figure 4: Mechanism of LV diastolic dysfunction due to the SFArich, lard-based HFD. 
investigated whether activation of Sirt 1 by nicotinamide mononucleotide (NMN) could reverse membrane phospholipid unsaturation and diastolic dysfunction in animals on a lard-based HFD. Administration of NMN increased Sirt1 activity and Scd1 expression, thereby reducing the membrane SFA/MUFA ratio and restoring $\mathrm{LV}$ diastolic function. Sirt1 regulates stearoyl-CoA desaturase (SCD-1) expression and thus contributes to maintaining a low SFA/MUFA ratio by promoting conversion of SFA to MUFA. The lard-based HFD impaired this counterregulatory mechanism by

\section{References}

1. Abdalrhim AD, Marroush TS, Austin EE, et al. Plasma osteopontin levels and adverse cardiovascular outcomes in the PEACE trial. PLoS One. 11(6): e0156965 (2016).

2. Sanchis-Gomar F, Santos-Lozano A, Pareja-Galeano H, et al. Galectin-3, osteopontin and successful aging. Clin Chem Lab Med. 54(5): 873-7 (2016).

3. Shirakawa K, Endo J, Kataoka M, et al. IL (Interleukin)-10STAT3-galectin-3 axis is essential for osteopontin-producing reparative macrophage polarization after myocardial infarction. Circulation. 138(18): 2021-35 (2018).

4. Shimatani K, Nakashima Y, Hattori M, et al. PD-1+ memory phenotype CD4+ T cells expressing C/EBPalpha underlie $\mathrm{T}$ cell suppressing Sirt1 expression, thus increasing the SFA/ MUFA ratio and worsening diastolic dysfunction.

\section{Conclusion}

Visceral fat obesity due to SFA overload accelerates the process of immune senescence. An anti-aging vaccine targeting senescent $\mathrm{T}$ cells has the potential to support productive aging. Excessive intake of SFA directly affects the fatty acid composition of cardiomyocyte membranes and induces diastolic dysfunction. Balanced intake of MUFA or activation of Sirt1 can ameliorate diastolic dysfunction due to SFA overload.

immunodepression in senescence and leukemia. Proc Natl Acad Sci. 106(37): 15807-12 (2009).

5. Shirakawa K, Yan X, Shinmura K, et al. Obesity accelerates T cell senescence in murine visceral adipose tissue. J Clin Invest. 126(12): 4626-39 (2016)

6. Shirakawa K, Endo J, Katsumata Y, et al. Negative legacy of obesity. PLoS One. 12(10): e0186303 (2017).

7. Yamamoto T, Endo J, Kataoka M, et al. Decrease in membrane phospholipids unsaturation correlates with myocardial diastolic dysfunction. PLoS One. 13(12): e0208396 (2018).

8. Yamamoto T, Endo J, Kataoka M, et al. Sirt1 counteracts decrease in membrane phospholipid unsaturation and diastolic dysfunction during saturated fatty acid overload. J Mol Cell Cardiol. 133: 1-11 (2019). 